\title{
REVISIONES
}

\section{Análisis de la discapacidad desde una mirada crítica: Las aportaciones de las teorías feministas}

\author{
Analysis of Disability from a Critical Perspective: \\ Contributions of Feminist Theories \\ Análise da discapacidade desde uma mirada crítica: \\ As contribuições das teorias feministas
}

\author{
Vanessa Gómez Bernal. ${ }^{a}$ \\ ${ }^{a}$ Universidad de Cádiz, España. C/Palacios, n $14,1^{\circ}$ C. C.P.: 11500 (El Puerto de Santa María). \\ Correo electrónico: v_g.bernal@hotmail.com
}

\begin{abstract}
RESUMEN
Al revisar el corpus teórico sobre la discapacidad nos damos cuenta de que el discurso predominante ha sido el que proviene de las disciplinas científicas propias del modelo médico-patológico. Por ello, se presenta un análisis crítico de la discapacidad aportando perspectivas situadas y contextualizadas política y socialmente que tienen en cuenta la voz y la vivencia de las personas implicadas. Las teorías feministas, enmarcadas en las Ciencias Sociales, resultan claves para desentrañar la construcción ideológica de los discursos que históricamente han servido para dominar y oprimir a las personas con discapacidad, y a su vez, para construir un sujeto anómalo y desviado que había que disciplinar.
\end{abstract}

Palabras clave: discapacidad, género, construcción social, teorías feministas, ciencias sociales.

\begin{abstract}
In reviewing the theoretical corpus on disability we realize that the predominant speech has come from scientific disciplines presenting a pathological medical model. Therefore, we present a critical analysis of disability providing prospects located politically and socially contextualized to take into account the voice and experience of the people involved. Feminist theories, framed in the Social Sciences, are keys to unraveling the ideological construction of the discourses that have historically served to dominate and oppress people with disabilities, and in turn, to construct a social subject abnormal and deviant to be disciplined.
\end{abstract}

Key words: disability, gender, social construction, feminist theories, social sciences.

\section{RESUMO}

Ao revisar o corpus teórico sobre a incapacidade dá-se conta de que o discurso predominante tem sido o que prove das disciplinas científicas próprias do modelo médico-patológico. Por isso, apresenta-se uma análise crítica da incapacidade contribuindo com perspectivas situadas e contextualizadas política e socialmente e que consideram a voz e a vivência das pessoas implicadas. Das teorias feministas, enquadradas nas Ciências Sociais, resultam chaves para desentranhar a construção ideológica dos discursos que, historicamente, têm servido para dominar e oprimir as pessoas com incapacidade e, assim, construir um sujeito anômalo e desviado que tem de ser disciplinado.

Palavras chave: incapacidade, gênero, construção social, teorias feministas, ciências sociais. 


\section{INTRODUCCIÓN ${ }^{1}$}

$$
\begin{array}{r}
{[\ldots] \text { y una noche sin mar ni pesadillas }} \\
\text { los Otros } \\
\text { esos Otros que inventamos }
\end{array}
$$

los Otros nos inventan nos recrean a su imagen y a su semejanza nos convencen de que al fin somos Otros

y somos Otros claro por suerte somos Otros.

(Benedetti, 1974: 79).

Con este artículo se pretende argumentar y fundamentar, con el aporte que las teorías y los paradigmas nos permiten, un posicionamiento teórico crítico para concebir la discapacidad y el género como campos de estudios confluentes en las Ciencias Sociales.

La representación social de las personas con discapacidad se deriva del discurso hegemónico. Este discurso se sustenta, puede decirse en términos generales, en el modelo médico de la sociedad que ve a las personas con discapacidad como incompletas, alejadas de la norma. Esto explica que se hayan ido desarrollando estudios científicos críticos -o al menos, alternativos-, en los que ha habido un punto de encuentro entre los estudios feministas y de la discapacidad desde las Ciencias Sociales, cuyo interés científico se basa en poner de manifiesto la construcción social de las inferioridades y de los discursos legitimadores de dominación.

Desde una perspectiva socioantropológica, un punto clave en este asunto es el establecimiento de una diferencia entre lo biológico y lo social. Para los conceptos de enfermedades y discapacidades ha sido fundamental poder separar la "enfermedad" de la “discapacidad". La separación entre "enfermedad" y "discapacidad” marca el cambio entre el "modelo médico-biológico" y un "modelo social" de la discapacidad, en cuyo enfoque nos hemos apoyado para enmarcar teóricamente este artículo, como a continuación se irá exponiendo.

Hasta muy recientemente, en la investigación y en la intervención profesional en el campo de la discapacidad ha prevalecido el enfoque individual, médico o patológico, centrándose la atención en el sujeto, en el déficit o en la alteración respecto de la norma, y sus consecuencias en el funcionamiento biológico, el desarrollo psicológico, el aprendizaje académico, la adaptación social, el desenvolvimiento en la vida cotidiana, etc. La investigación orientada desde perspectivas sociopolíticas (interaccionismo simbólico, estructuralismo, constructivismo) ha aportado un importante avance en la comprensión de la discapacidad y su teorización, llegando a conformar lo que se viene denominando “modelo social de discapacidad" (López González, 2007: 139).

Por tanto, este artículo se va a centrar en analizar la conjugación de dos constructos que configuran relaciones de poder: discapacidad y género. En primer lugar, se abordará la conceptualización de la discapacidad en el marco de las perspectivas constructivistas y de la teoría crítica en Ciencias Sociales, que nos pueden permitir esclarecer la herencia

\footnotetext{
Agradezco la revisión y las apreciaciones sobre este artículo a Rosa Vázquez Recio y a Francisco Vázquez García de la Universidad de Cádiz.
} 
científica-ideológica que hemos recibido para comprender dicho fenómeno de manera unicausal, biologicista e individualizante. En segundo lugar, el análisis de las relaciones entre género y discapacidad se hace desde una mirada crítica feminista. Pese a los ataques y el desprestigio que desde algunas perspectivas científicas se le proporciona, con la pretensión de monopolizar "lo objetivo" en las investigaciones sociales, las aportaciones feministas en las investigaciones sobre la discapacidad son clave para la comprensión del fenómeno objeto de estudio en este trabajo.

\section{LA DISCAPACIDAD RE-CONOCIDA COMO CAMPO EN LAS CIENCIAS SOCIALES}

Hay que saber perderse para trazar un mapa, salir de los caminos trillados, vagar: deambular por las encrucijadas, abrir senderos a través de los mieses o el desierto, penetrar en callejuelas sin salida, asumir que todo camino recorrido sin mapa es caótico [...]Y hay que subvertir la ley: apearse de todo lo dicho o lo sabido... (Ibáñez, 1992: 355).

Es relativamente reciente en el tiempo la conceptualización de la discapacidad desde paradigmas propios de las Ciencias Sociales. Esto ha permitido abrir nuevos horizontes para pensar la discapacidad, y re-conocerla como un campo científico, político y social con entidad propia. Es por ello que en estas líneas se asume y se concibe la discapacidad bajo la noción de campo, siguiendo las aportaciones del sociólogo Pierre Bourdieu (2007 [1998], 1991; Bourdieu y Wacquant, 1995), y de las revisiones de las lecturas de la investigación de Soledad Torres Dávila (2004). Asumirla en este sentido significa concebir la discapacidad, en tanto que campo científico y político, en un sentido relacional. Bourdieu sostiene que un campo es un espacio social, un microcosmos, un universo, un espacio práctico de la existencia cotidiana, que de acuerdo a leyes propias son relativamente autónomos (Bourdieu y Wacquant, 1992). Bajo esta perspectiva, entonces, podemos asumir la discapacidad como el espacio social que, siendo común a muchas personas, no es generalizable ni universal, y es así porque define las experiencias vitales de un grupo de personas, ya sean pacientes, profesionales de la medicina, familiares, instituciones, etc., asumidas y problematizadas de acuerdo a ejes como la "raza", la religión, la clase, el género, la ubicación geográfica, las condiciones de salud, entre otros, en tanto que campo tener discapacidad no excluye estar inmerso/a en otras situaciones sociales.

Como indica Torres Dávila (2004: 19), la discapacidad como campo hace referencia a un conjunto de relaciones, entendidas como las determinaciones que a partir de una propia lógica definen sujetos (personal sanitario, instituciones, padres, madres, voluntarios/as); posiciones (médico/a-paciente, niño/a-padre, niño/a-madre), y disposiciones o actitudes, esquemas, prácticas y discursos. En la discapacidad existe una multiplicidad de posiciones de los diferentes sujetos, no es objetivamente igual detentar la posición de paciente, de familiar, o de profesional de la medicina, porque socialmente se asignan reconocimientos, cualidades, obligaciones y derechos distintos en la medida que depende del sistema de diferenciaciones.

A lo largo de este trabajo aparecerá el concepto de "raza" entrecomillado, para expresar el propio cuestionamiento hacia la interpretación que de tal concepto se puede hacer. Se pretende mostrar la crítica de la utilización esencialista y determinista que se ha hecho de este concepto desde muchos espacios científicos. 
Si la discapacidad asumida como un campo implica una serie de sujetos, posiciones y disposiciones, las relaciones que se establecen en su interior son de fuerza y de luchas, ya que cada posición y cada sujeto posee diferentes capitales (físico, económico, cultural, simbólico), que permiten a cada uno ejercer un determinado poder reconocido socialmente.

La discapacidad como campo entonces, promueve diversos poderes desde los sujetos, las prácticas y los discursos, que pueden buscar mantener el campo con su dinámica propia, transformarlo o simplemente resistirlo. Sin embargo, estas estrategias están condicionadas por las percepciones que los sujetos tienen sobre la discapacidad como campo y que a su vez las define, da forma y contenido a sus prácticas y discursos (Torres Dávila, 2004: 20-21).

La interrelación de los ejes de clase social, género, religión, pertenencia cultural, sexualidad, etc., que en la discapacidad apuntan a la diversidad en la cotidianidad de las experiencias, actúan como filtros a través de los cuales no sólo se matizan las experiencias vitales en torno a las discapacidades, sino que demuestran la complejidad de las relaciones sociales existentes en la discapacidad como campo (Torres Dávila, 2004).

Por otra parte, desde inicios de los años 90, el campo científico de la discapacidad se ha visto inundado de numerosas publicaciones teóricas, artículos y libros, producidas por académicas/os del Reino Unido y de EEUU, que por medio de unas contundentes y radicales críticas al modelo médico, plantean un modelo social de la discapacidad (Albrecht, Seelman y Bury, 2001; Barnes, Mercer y Shakespeare, 1997; Bradley, 1995; Corker, 1998; Davis, 1997; Imrie, 1997; Marks, 1999, 1997; Moore, Beazley y Maelzer, 1998; Oliver, 1992, 1990).

La discapacidad ha sido tradicionalmente considerada como un problema que afecta únicamente a los individuos particularmente, como un problema derivado de las condiciones de salud individuales y de deficiencia de algunos sujetos cuyos cuerpos se apartan de los cánones de la normalidad y que, por ende, se transforman en objeto de estudio e intervención de la medicina, la psicología y la pedagogía.

En esta perspectiva, anclada en los desarrollos funcionalistas, la discapacidad es pensada en términos de tragedia personal o de desviación social (Rosato et al., 2009: 90). Conceptualizar la discapacidad como desviación social implica asignarle una identidad y condición social devaluadas, que se convierten en una faceta central de la existencia y que afectan a la estructura psíquica del sujeto. Con ello, la persona con discapacidad es condenada a una especie de ciudadanía devaluada, considerada como una menor de edad permanente que, en el caso de tener derechos, tiene suspendido su ejercicio o requiere de la tutela de otros para ejercerlos. Mike Oliver (1998) propone que los valores principales del ideario liberal son las responsabilidades individuales, la competencia y el trabajo. Quienes son incapaces de cumplir con estos ideales, imperantes en nuestra sociedad, son calificados como desviados por su incapacidad para asumir los roles socialmente adjudicados. Es por ello que se etiqueta al sujeto como "discapacitado" en un contexto que expone cuáles son las pautas de comportamiento y funcionamiento valoradas socialmente.

Se propone entender aquí, por lo tanto, la discapacidad

como una producción social, inscripta en los modos de producción y reproducción de una sociedad. Ello supone la ruptura con la idea de déficit, su pretendida causalidad biológica y consiguiente carácter natural, a la vez que posibilita entender que su significado es fruto de 
una disputa o de un consenso, que se trata de una invención, de una ficción y no de algo dado. Es, también, una categoría dentro de un sistema de clasificación y producción de sujetos (Rosato et al., 2009: 92-93).

\subsection{LA CONSTRUCCIÓN DE LAS CATEGORÍAS BINARIAS EN EL DISCURSO CIENTÍFICO}

La organización social se asienta en un conjunto de normas consideradas naturales, normales, inevitables, necesarias... En este sentido, un período de tiempo especialmente significativo en nuestra historia es la Modernidad de donde, como explica Arnau Ripollés (2004), hemos aprendido que el ser humano tiene dignidad intrínseca, y que ésta es la característica más propia que le dota de humanidad, y que es consustancial a su Ser. Con ello, la dignidad se constituye, al mismo tiempo, como la característica más universal y "digna de universalizarse" de todas las posibles. La dignidad no es respetada, ni reconocida para todo ser humano, obviando otra peculiaridad del ser humano que igualmente le dota de "humanidad", y que también le es consustancial según Arnau Ripollés (2004); nos referimos a la diversidad humana. Es por todo ello por lo que se hace hincapié en posturas menos ortodoxas, y más críticas, ya que "en nuestro afán de universalizar hemos dejado en los márgenes a muchos grupos y colectivos en la medida en que se salían de la norma" (Arnau Ripollés, 2004: 1).

Si bien el feminismo fue de esos movimientos que cuestionó la universalidad del sujeto moderno cuyo paradigma es el hombre, blanco, heterosexual y con privilegios de clase en diferentes épocas, también reprodujo esta misma universalidad cuando se pensó que su sujeto colectivo era "la mujer", de forma homogénea; todo ello definido desde posiciones de privilegios de clase, "raza" y sexualidad (Curiel, 2011: 199). Las feministas negras fueron las primeras en cuestionar esa universalidad y lo hicieron no sólo desde las teorías, sino desde la militancia política. De este modo, estaban construyendo otro conocimiento y un proceso de descolonización epistemológica y política.

La discapacidad como producción social supone tener en cuenta cuál es el contexto socio-histórico en el que nos situamos, de qué manera está permeado el conocimiento por valores culturales, por normas sociales, por el lenguaje... Obviamente no se trata de un concepto unívoco, y es necesario enmarcarlo en una realidad histórica y sociocultural concreta. Por todo ello, es fundamental analizar la construcción de categorías binarias que cobran una especial relevancia en las personas con discapacidad: normal/patológico, salud/ enfermedad, válida/inválida, masculino/femenino...

Partir de la idea de la normalidad como construcción social e histórica implica, en primer lugar, desnaturalizar los modos únicos y hegemónicos en que ha sido entendida. Por otro lado, también nos permite reconocer los procesos sociales, históricos y políticos que dieron lugar a la idea de la normalidad única como parámetro de clasificación y de prescripción de modos de ser y de estar en el mundo.

En esta línea argumental, es interesante analizar dicotomías como la de salud/ enfermedad. La salud es un fenómeno social como lo es biológico. La salud de cualquier población viene determinada por aspectos sociales. Los estándares de salud varían de un lugar a otro y con el paso del tiempo. Lo que consideramos "estado de salud" guarda una estrecha relación con la desigualdad social. A pesar de que el significado dado al fenómeno de la enfermedad ha variado entre las diferentes culturas históricas, su importancia social es el resultado de un factor común a todas ellas: la amenaza que representa para el ser humano y su sociedad (Rodríguez, 1987). Según Rosenberg (1989), 
la enfermedad es "un acontecimiento biológico", pero también "un peculiar repertorio generador de construcciones verbales que reflejan la historia intelectual e institucional de la medicina, un aspecto de la política pública y la legitimación potencial de ésta, un elemento potencialmente definidor de roles sociales, una sanción de normas culturales y un elemento estructurador en las interacciones médico-paciente". Y concluye apuntando que "de alguna manera, la enfermedad no existe hasta que hemos acordado su existencia, al percibirla, nombrarla e intervenir sobre ella" (1989: 1-15). Las enfermedades que etiquetamos como tales no constituyen, según dicho acercamiento teórico, entidades naturales transhistóricas que se mantienen en el espacio y el tiempo, sino que también tienen que ser entendidas como producciones sociales que se desarrollan en contextos socioculturales concretos.

En esta coherencia de ideas se propone pensar que la noción de normalidad social no puede pensarse sino en términos de ideología, y en este sentido se acuña el concepto de "ideología de la normalidad" (Rosato et al., 2009). El hecho de que las distintas teorías de la discapacidad que la homologan al "déficit" operen más allá de la conciencia naturalizando sus predicados, constituye un rasgo particular del trabajo ideológico de la ideología de la normalidad. La ideología de la normalidad, a través del discurso médico-pedagógico, genera todo un desarrollo conceptual y metodológico tendiente a instalar una supuesta causa biomédica de la inteligencia y de las capacidades que, por causas naturales, estarían distribuidas en forma desigual entre los sujetos.

En el caso de las personas con discapacidad, la ideología de la normalidad no sólo las define por lo que no tienen: su falta, su déficit, su desviación, su ausencia y su carencia, sino que también y simultáneamente confirma el "estar completo" de las no discapacitadas, que suelen ser igualadas y asignadas a la categoría de normales. Esta lógica binaria (normal/ a-normal) se asienta sobre el convencimiento del valor de la normalidad: está bien "ser normal" y, si alguien no lo es, le resulta imperativo hacer los tratamientos de rehabilitación necesarios para acercarse lo más posible a ese estado o esa condición (Balza, 2011: 99). Ésta es una idea constante que construye la sensación de que lo normal es algo obvio, que no es necesario explicar, porque para el sentido común es una categoría demarcatoria entre quienes cumplen con sus requisitos y quiénes no. Esta normalidad, ni fechada ni localizada y que no es objeto de ninguna clarificación de fondo, es proclive a funcionar como sinónimo de natural. En este sentido, "naturalizar la normalidad y los procesos de exclusión que a partir de ella operarían implica echar abajo la posibilidad misma de la problemática puesta en acto, basada en el carácter social, histórico, relativo de la exclusión, y por tanto en su solución posible" (Karsz, 2004: 188).

Las consecuencias de la enfermedad, definidas como discapacidad, fueron interpretadas y clasificadas aplicando el modelo de enfermedad tradicionalmente utilizado en las ciencias de la salud. Así se desarrollaron sistemas de clasificación de los déficits que, siguiendo los mismos principios que los utilizados en las clasificaciones de las enfermedades, perseguían establecer la taxonomía de las discapacidades. Se diseñaba de esta manera una serie de medidas terapéuticas y compensatorias que tenía destinatarios individuales. Este enfoque perpetuaba la estigmatización de las personas con discapacidades, al considerar que era responsabilidad de cada individuo conseguir su plena adaptación al medio.

El modelo médico-biológico, debido a la unidimensionalidad de sus planteamientos, fue reconducido a través de una perspectiva social. En 1958 el sociólogo Talcott Parsons sostenía al respecto que el estado normal del ser humano en las sociedades occidentales desarrolladas era la denominada "buena salud", por lo que la enfermedad y, por tanto, las 
deficiencias debían ser tratadas como desviaciones de la normalidad. Parsons acuñó la expresión "conducta de enfermedad" (comportamiento ante la situación de enfermedad). En su obra El sistema social (1982 [1951]) hace referencia a las relaciones existentes entre enfermedad y anormalidad. Así, considera que la enfermedad tiene que ser definida como una forma de conducta desviada, es disfuncional y va en detrimento del cumplimiento de objetivos sociales, obstaculizando el funcionamiento correcto de la sociedad. Para este autor enmarcado en la corriente positivista, la desviación es real, existe en la experiencia objetiva y remite al comportamiento que se encuentra por fuera del sistema normativo (Cit. en Martín Jorge y Soriano Rovira, 2008: 99-100).

Erving Goffman (2001 [1963]) cuestionó esta visión ortodoxa según la cual los problemas asociados con la discapacidad eran el resultado directo de las deficiencias individuales. Para este autor, las situaciones de dependencia se generaban a partir del proceso de estigmatización en el cual los profesionales de la rehabilitación jugaban un papel fundamental. El estigma aparece como una característica de los sujetos que inhabilita para la plena aceptación social (Goffman, 2001 [1963]).

En este asunto, un autor ineludible para comprender el proceso de construcción de determinadas categorías es sin duda Michel Foucault. En su obra Historia de la sexualidad (2009 [1978]) nos acerca a las prácticas de disciplinamientos y de control como formas de inserción controlada de los cuerpos en el aparato de producción a través de lo que denominó biopoder. Este biopoder se expresa a través de dos técnicas. Por un lado, la anátomo-política, tecnología individualizante, que se centra en el control del cuerpo por medio de instituciones como la cárcel, el manicomio, el hospital o la escuela, con el fin de anatomizarlo, es decir, de producir cuerpos dóciles y fragmentados para su regulación. Por otro lado, la bio-política, que se ocupa de la regulación del "cuerpo-especie" o de la población por medio del control de la natalidad, la fecundidad, la morbilidad, etc. El punto de articulación entre ambas técnicas radica en el control de la sexualidad como mecanismo de producción disciplinante del cuerpo y las regulaciones de poblaciones. En el caso de las personas con discapacidad confluyen las dos técnicas del biopoder: el control sobre el cuerpo de los sujetos, y el control sobre la reproducción de la especie subhumana que supuestamente supone la discapacidad.

Por otra parte, el binomio normal/patológico se ha ido transformando a lo largo de la historia desde un plano descriptivo a un plano prescriptivo, es decir, no sólo dice o describe cómo son las poblaciones sino cómo deben ser. Así, no sólo se comienza a trabajar sobre la idea de clasificar lo "normal" distinguiéndolo de lo "anormal", sino que se estructuran prácticas y saberes encargados de normalizar a extensos grupos de sujetos que no cumplan las características deseables (Foucault, 2009 [1978]). Por tanto, una pregunta relevante en este tema, además de quién clasifica a quién, y según qué parámetros y qué relaciones habilitan esta clasificación, es qué procesos de naturalización y legitimación han hecho posible esta situación. Se trata de una "ideología de la normalidad" como sostiene Susan Wright, "una ideología hegemónica se torna tan naturalizada, dada por hecho y "verdadera" que las alternativas están fuera de los límites de lo imaginable" (1998: 14-15).

Siguiendo estas interpretaciones foucaultianas, detectamos una ruptura entre modelos previos que entienden la perfección humana como una naturaleza dada por Dios y un modelo moderno, ya médico-científico, que permanece privilegiado en la cultura occidental. Una de las aportaciones claves desde esta perspectiva es la del filósofo Georges Canguilhem (2005 [1966]) con sus investigaciones sobre la cuestión de la normalidad y la patología. Canguilhem investiga cómo la modernidad postcartesiana categoriza y establece claros 
límites de separación entre lo que se considera normal y el otro patológico o anormal. En esta línea de investigación, este autor va a examinar la figura del monstruo en tanto que representación histórica occidental de lo anormal o lo patológico (Canguilhem, 2005 [1966]). La construcción y el mantenimiento de la distinción entre una corporalidad normal y otra patológica van a permitir que unos cuerpos sean catalogados como deficientes y, en consecuencia, sean discriminados socialmente.

Deconstruir el modelo naturalizado de la discapacidad heredero del paradigma moderno va a ser un elemento clave en la crítica feminista, lo que va a permitir construir otros modelos de la discapacidad no enmarcados ya en la oposición normal/patológico. Las dos prácticas que propone Thomson (2005) para desmontar el sujeto discapaz naturalizado, recogen el núcleo de la estrategia crítica que se ha propuesto desde los estudios feministas de la discapacidad. La primera es la de eludir las categorías del diagnóstico médico para pensar la discapacidad. La segunda estrategia crítica que utiliza la autora es la de cuestionar las asunciones culturales del uso del lenguaje: se critica el uso de términos tales como "deformidades" o "anormalidades". Lo que propugna es no caer en definiciones esencialistas de la discapacidad en tanto que se interpreta como una encarnación inferior del ser humano. Paralelamente, tener en cuenta cuál es el paradigma ideológico desde el que se clasifica a los sujetos con discapacidades es clave para despatologizar la discapacidad.

Las personas con discapacidad son portadoras de una singularidad en virtud de la cual han sido catalogadas de determinada manera, y han sido objeto de ciertas prácticas que han transformado su singularidad en una diferencia marcada negativamente y sobre la que se edifica su experiencia vital. Debido a dicha marca, las personas con discapacidad han sido definidas mediante conceptos que son el polo negativo de categorizaciones dicotómicas: son personas discapacitadas (sin capacidad), anormales (sin normalidad), enfermas (sin salud), dependientes (sin independencia), asexuadas ( $\sin$ sexo), en suma, son personas defectuosas (Rodríguez Díaz y Ferreira, 2010: 295).

Es evidente que la pretensión normalizadora de la parte dominante de la medicina y la psiquiatría, facilitó la creación de nuevos espacios en los que enumerar y clasificar a las personas. No es que una determinada clase de sujetos ya existente comenzara a ser reconocida por administradores o por científicos expertos en la naturaleza humana, sino que esa clase de individuos se inventa, se construye, al mismo tiempo que la propia clase o categoría es formulada.

Las clases, especies o variedades "naturales" (desde los electrones a los distintos rangos taxonómicos del reino animal, vegetal o mineral) son indiferentes, no cambian según la manera en que sean nombrados y no son afectadas por las prácticas culturales, pero las clases humanas son interactivas, su manera de ser y de actuar, su subjetividad y sus acciones no son independientes de cómo son descritos y clasificados (Huertas, 2011: 446).

Esto es lo que Ian Hacking $(2007,1994)$ llama el "efecto bucle" de las clases humanas: "las interrelaciones entre la gente y las formas en que ésta es clasificada. Las personas tienden a conformarse, a permanecer e incluso a crecer, en el ámbito clasificatorio en el que han sido descritos o diagnosticados" (Cit. en Huertas, 2011: 447). Hacking sigue a Erving Goffman cuando advierte que ciertas categorías de individuos son creadas; no existen más que en el momento de ser definidas y estudiadas por algunas disciplinas científicas. Sin embargo, "esas categorías, esas etiquetas, tienen efectos sobre las personas clasificadas; 
unas veces de manera directa, emanado de su propio conocimiento de haber sido catalogadas. Otras veces, el efecto bucle es indirecto, sobre todo cuando las clasificaciones son incorporadas al funcionamiento de las instituciones" (Cit. en Huertas, 2011: 447).

\subsection{SOBRE LA INCORPORACIÓN DE LOS DISCURSOS HEGEMÓNICOS DE LA DISCAPACIDAD}

La ciencia médica se ha constituido en el enlace, en el nivel del saber, entre la disciplina de los cuerpos individuales y la regulación de las poblaciones. La medicalización de los cuerpos se ha convertido en una de las herramientas utilizadas para el control de las personas (Rodríguez Díaz y Ferreira, 2010). Hay que recordar el carácter "sagrado" que tiene en nuestra sociedad el conocimiento científico, cuyos saberes son admitidos como algo incuestionable y de una categoría superior a los saberes más intuitivos o populares. La eficacia se deriva de la imposición científica de normas políticas, de sujeción de los cuerpos, de la "normalización" de la existencia en virtud de dictámenes científicos (Rodríguez Díaz y Ferreira, 2010: 297). En este sentido, son las ciencias biotecnológicas, la genética y las tecnociencias, las que se erigen como fuentes absolutas de "Verdad". Como indica Braidotti,

el ser humano ha sido subsumido en relaciones globales de intimidad, complicidad y proximidad con fuerzas de tipo inhumano y poshumano, complejos científicos, industriales y militares, redes de comunicación global y procesos de mercantilización e intercambio a escala global. Estas relaciones constituyen complejas redes de interfaz con las tecnologías que han hecho de la "Vida" su mecanismo de control favorito (2005: 321).

Siguiendo el análisis foucaultiano, y como indicábamos anteriormente, las tecnologías del poder se dividen en disciplinas del cuerpo (anátomo-política) y regulaciones de la población (bio-política). El poder estatal, para gobernar una sociedad en fase de explosión demográfica e industrialización, tuvo que, en primer lugar, reconocer lo particular mediante una primera adaptación de los mecanismos de poder (disciplina, vigilancia, adiestramiento), que surgen entre el siglo XVII y XVIII en instituciones como la escuela, el hospital, el cuartel o la fábrica. Nuevos poderes laterales a la justicia cristalizarían en instituciones de vigilancia (la policía) y de corrección, -psicológicas, psiquiátricas, criminológicas, médicas y pedagógicas-, dando paso así a la edad de la "ortopedia social", con un tipo de poder disciplinario penal (Foucault, 2009 [1979]). Las formulaciones de Foucault en torno a las estrategias políticas de normalización son especialmente valiosas para comprender los procesos de desigualdad social con referencia a la discapacidad y a los procesos de inscripción de la desigualdad de género.

Sobre los cuerpos se inscriben, tanto mediante adiestramiento como a través de significaciones con efectos performativos, normas de regulación de la existencia. Se trata de prescripciones normativas en las que lo "normal" cumple la doble función de señalar una regularidad estadística (el caso mayoritario) y una prescripción moral (lo bueno y adecuado), pudiendo darse el caso de que la prescripción sea causa de la regularidad más que a la inversa (Rodríguez Díaz y Ferreira, 2010: 290-291). En el caso de las personas con discapacidad, esa naturalización del cuerpo hace que las directrices normalizadoras sean estipuladas por la ciencia médica, de modo que se establece una indisociable vinculación entre discapacidad y enfermedad. Ello nos conducirá a evaluar la condicionalidad de dicha norma de salud, la cual, lejos de un criterio de validez universal, está sujeta a las demandas 
de funcionalidad y eficiencia que los contextos sociales, políticos, históricos y culturales imponen sobre el organismo humano (Rodríguez Díaz y Ferreira, 2010). Se justifica dicha toma de posición al tomar en consideración la propuesta foucaultiana de que nuestra experiencia está regulada por aparatos de saber-poder, discursos expertos que discriminan sobre lo "verdadero" y lo "falso", pero puestos al servicio del poder político para dictaminar las normas/verdades que habrán de ser cumplidas como ejercicio de sometimiento al poder.

La importancia de la "incorporación" de las prácticas y las lógicas de dominación y su inscripción en los mismos cuerpos de las personas con discapacidad, ha sido analizada por Ferrante y Ferreira (2008) haciendo referencia a la noción de habitus del sociólogo Pierre Bourdieu. La obra de Bourdieu constituye una producción amplia dentro de la corriente constructivista y estructuralista en las Ciencias Sociales. Como el autor explica, "por estructuralismo entiendo la afirmación de que existen, en el mundo social mismo, y no sólo en los sistemas simbólicos como el lenguaje, el mito, etc., estructuras objetivas independientes de la conciencia y de la voluntad de los agentes y capaces de orientar o de restringir sus prácticas y sus representaciones" (Bourdieu, 1987: 147). En cuanto al constructivismo lo explica así: "entiendo por constructivismo la afirmación de que existe una génesis social de los esquemas de percepción, de pensamiento y de acción que son constitutivos de lo que llamo habitus, por una parte; y por otra de las estructuras sociales, particularmente de lo que llamo campos o grupos, así como también de lo que ordinariamente suelen llamarse clases sociales" (Bourdieu, 1987: 147). Así pues, el concepto de habitus se constituye en un instrumento de regularidad de las conductas, haciendo predecibles ciertas prácticas, ya que el habitus hace que los agentes sociales se comporten de un modo determinado en ciertas circunstancias. Por tanto, las representaciones de los agentes varían según su posición en la red social y según su habitus, el cual conforma un conjunto de esquemas de apreciación y de percepción. El habitus produce prácticas y representaciones que están disponibles para la clasificación, pero que no se perciben en lo inmediato, salvo por los agentes que poseen el código.

Como plantea el sociólogo Miguel A. V. Ferreira (2010: 47), el habitus es una estructura de predisposiciones que confiere a la persona su "competencia" para desenvolverse en el mundo. Tal estructura, heredada del grupo de pertenencia, delimita objetivamente las opciones posibles a disposición del agente pues incorpora las predisposiciones cognitivas, perceptivas, interpretativas, etc., que definirán sus capacidades como tal. Por tanto, el habitus sólo tiene sentido en su puesta en práctica, y en ella y como consecuencia, de los efectos prácticos que suscita, el agente lo va modificando al apropiárselo. "Estamos inevitablemente constreñidos de antemano a ser capaces de pensar, actuar y sentir de determinadas maneras (e incapacitados para hacerlo de muchas otras), pero esa constricción es, justamente, la base sobre la cual podemos adoptar elecciones particulares muy dispares" (Ferreira, 2010: 48).

Ser una mujer con discapacidad implica la pertenencia a un grupo de referencia, proveedor y portador de un habitus específico y concreto. Las predisposiciones adquiribles a partir de él están determinadas de antemano por el sentido asociado al hecho de poseer una discapacidad y por las prácticas vinculadas a dicha posesión. El sentido socialmente legítimo de la discapacidad proviene de la ciencia médica, que es la que suele determinar y estipular los estándares de funcionamiento "normal", generando ciertos parámetros cuantitativos de validez presuntamente universal para catalogar a los cuerpos humanos como sanos o enfermos. Cualquier mujer con discapacidad, bien desde su nacimiento o bien desde el momento en que adquiere dicha condición, experimenta la clara evidencia de que su 
condición supone una limitación en sus posibilidades de desenvolvimiento, porque su cuerpo es imperfecto, inadecuado para lo que se considera habitual. Esto se ha mostrado como una condición natural de su existencia, de la persona con discapacidad, como la evidencia incuestionable de una corporalidad, objetivamente imperfecta (Ferreira, 2010: 49).

\section{DISCAPACIDAD Y GÉNERO COMO CATEGORÍAS DE ANÁLISIS CRÍTICAS}

Renunciar a domesticar la vida y apostar por el rol de la pasión y de la emoción en la estructuración social como alternativa a la razón y a la eficacia extremas, es abrir corredores por los que pueda circular una nueva energía capaz de redimir a lo instituido como agencia al servicio de todo dominio (Navarro, 2004: 181).

A continuación vamos a presentar el aporte de las teorías feministas en las Ciencias Sociales para el análisis del fenómeno de la discapacidad y, en concreto, de su relación con el género.

\subsection{LAS TEORÍAS FEMINISTAS EN LOS ESTUDIOS DE LA DISCAPACIDAD}

El análisis de la discapacidad a través de una perspectiva de género es un campo novedoso y de interés reciente y creciente. Esto se debe en parte a que las mujeres con discapacidades han estado invisibilizadas tanto en los estudios de género como en las investigaciones sociales sobre la discapacidad; esto nos remite a la idea de la "múltiple opresión". Ser mujer con discapacidad marca una trayectoria de múltiple discriminación y añade barreras que dificultan el ejercicio de derechos, la plena participación social y la consecución de objetivos personales.

La discapacidad condiciona la forma que tienen las personas de relacionarse con el mundo, configurándose como un factor determinante en la construcción de su identidad, al igual que otros elementos de sus vidas como el género, la edad, la clase social, etc. Así pues, la discapacidad puede ser considerada, sin lugar a dudas, una variable que contribuye a la construcción de la identidad de los individuos. No obstante, el modo en que afecta a mujeres y a hombres es diferente, ya que interactúa con las incorporaciones del género y modula alguno de los factores que intervienen en la construcción de la identidad de las mujeres con discapacidad. Las causas se encuentran en la base de la representación mental y simbólica, fruto de los estereotipos y de los roles existentes, que son diferentes para hombres y mujeres en nuestra sociedad (Shum y Conde, 2009: 130). Como señala Tom Shakespeare (1998: 205-209), los estereotipos sexuales refuerzan los prejuicios sobre la discapacidad, ya que en el caso de mujeres con discapacidad, la dependencia, la vulnerabilidad y la debilidad son las asociaciones en la cultura patriarcal. Por consiguiente, las mujeres con discapacidades se representan de una manera especialmente negativa y pasiva. Discapacidad y género se tornan, pues, en variables determinantes en la interacción social que las personas con discapacidades establecen con el mundo "no-discapacitado".

En el intento de buscar una alternativa crítica a los planteamientos dominantes sobre la comprensión de un fenómeno social como lo es la discapacidad, los estudios feministas jugaron un papel clave. Los estudios feministas de la discapacidad surgen en los años 90 en el ámbito anglosajón. La crítica feminista de la discapacidad no se distingue porque su 
objeto de análisis sean las mujeres con discapacidades, sino porque estudia la discapacidad desde un paradigma teórico propio de los estudios de género, con una perspectiva crítica del sistema de género y de la conceptualización de la opresión. De este modo, la crítica feminista de la discapacidad fundamenta sus análisis en las propuestas de los estudios feministas, atendiendo a las estructuras de dominación y discriminación que conforman el orden social. Tal y como diversas autoras señalan, los estudios feministas de la discapacidad sitúan la experiencia de la discapacidad en el contexto de los derechos y las exclusiones, insistiendo en un modelo social de la discapacidad, frente al modelo médico imperante hasta los años 70 (Balza, 2011: 57). El modelo médico se define por ser un modelo binario, que opone al sujeto normalizado un sujeto defectuoso o anormal, aquel que no cumple con los estándares de la mayoría normalizada. Por ello, una de las apuestas fundamentales de la crítica feminista de la discapacidad es la de desnaturalizar la discapacidad, investigando para ello el modo en que históricamente se ha ido construyendo el paradigma de la normalidad y el sujeto desviado. En este sentido, entender la discapacidad como una producción social implica una visión socio-política de la discapacidad (Barton, 1998; Rosato et al., 2009), con el objetivo de dar cuenta no sólo del proceso de su producción, sino también de la injusticia económica y simbólico-cultural a las que están sometidas las mujeres discapacitadas.

Hasta hace bien poco tratar la intersección del género y la discapacidad parecía más bien ser fruto del capricho teórico que de una necesidad real de denunciar años de ocultamiento y exclusión de las mujeres con discapacidad. La lucha por los derechos de las mujeres con discapacidad ha implicado necesariamente colocarse unas "gafas con doble aumento" (Cózar Gutiérrez, 2011: 12), que diera la perspectiva más acertada acerca de la dimensión que cobra la múltiple discriminación. Sin embargo, debe quedar claro que el hecho de que el colectivo sea activo y reaccione eficazmente ante situaciones discriminatorias relacionadas con la discapacidad, no lo hace más sensible a otro tipo de discriminaciones, como por ejemplo, la sexista.

Por otra parte, en los últimos años se ha producido un interesante giro en el modo de abordar la cuestión de la discapacidad. Este redimensionamiento se traduce en la aparición de estudios que analizan, desde una nueva perspectiva, las barreras que discapacitan a los individuos. Estas barreras tienen una dimensión histórica y social por lo que se hace necesario entrar a conocerla y criticarla con el objetivo de evidenciar de qué manera la opresión se configura en relación con el sistema de valores en los que se fundamenta nuestra sociedad. Las teorías feministas nos aportan el análisis sobre el papel que juega la discapacidad como categoría de análisis social en el modelo capitalista global y su conexión con otras diferencias sociales como la pertenencia a una etnia, la clase social, el género, la orientación sexual, etc. Para abordar el enfoque de la discapacidad desde una perspectiva feminista, es necesario realizar una serie de precisiones en torno a dos conceptos fundamentales: el concepto de discapacidad y las relaciones patriarcales. Tanto el cuerpo femenino como el cuerpo con discapacidad están atravesados por discursos culturales que lo sitúan en el ámbito de lo otro en relación con la normatividad impuesta por el cuerpo masculino, blanco, propietario, heterosexual y sin discapacidad.

Las estructuras de clase, "raza", género y sexualidad no pueden tratarse como variables independientes porque la opresión de cada una está inscrita en las otras, es constituida por y es constitutiva de las otras. La confluencia de estos dos constructos sociales, género y discapacidad -entre otros- sobre las mujeres con discapacidad, las sitúa en una "región gris" (Cózar Gutiérrez, 2011: 23), donde las situaciones de exclusión y discriminación 
son complejas y las estrategias de solución difieren de las ensayadas por las mujeres sin discapacidad. En ocasiones, las mujeres con discapacidad han seguido el camino, no exento de dificultades, de identificarse con los roles tradicionales establecidos a través del patriarcado, con el objetivo último de integrarse en la mayoría de manera acrítica.

Es de especial mención aquí, en el valor que tienen los estudios de género de la discapacidad, el concepto de interseccionalidad, que se manifiesta como una interacción entre la teoría feminista, el feminismo negro y la teoría postcolonial a finales de la década de los 90. La interseccionalidad es una teoría para analizar la producción de poder que se centra fundamentalmente en las minorías. Concepto de imbricación, interseccionalidad o matriz de dominación como lo llama Hill Collins (1998), que articula múltiples opresiones de "raza", clase y sexo. Por tanto, las estructuras de clase, "raza", género y sexualidad son concebidas como "variables dependientes" porque cada una se inscribe en las otras y es constitutiva de y por las otras. Las dos primeras olas del feminismo ignoraron a las mujeres con discapacidad, dejándolas a ellas fuera de los debates feministas. Las mujeres con discapacidad quedaban relegadas al ámbito de las organizaciones de personas con discapacidad, y eran consideradas objetos de cuidado. Este aspecto entraba en conflicto con las mujeres sin discapacidad en su camino de abolir sus obligaciones de cuidado y en la lucha por sus derechos individuales e independencia. Se consideraba que sus demandas no podrían integrarse en las del resto de mujeres sin discapacidad.

La diversidad de las mujeres se ha ignorado a favor de un modelo hegemónico, único y singular. Sobre esta cuestión, Bell Hooks (2005) nos cuenta en su artículo "Racismo estético: Alisando nuestro pelo", cómo el ritual de transición a la pubertad de las niñas afroamericanas en Estados Unidos se realiza a través del planchado del cabello, y cómo el cabello fino y dócil de cualquier mujer negra es un plus de belleza que viene marcado por cánones estéticos que parten de un modelo blanco difícilmente alcanzable para quien no lo es. El significado simbólico de la niña negra con el cabello planchado podría asimilarse a la de cualquier niña con discapacidad que se ve empujada a domesticar un cuerpo que tampoco cumple con la normatividad impuesta, del cuerpo capaz por antonomasia.

Otra referencia importante en este sentido es Sojourner Truth, una esclava liberada en EE.UU en el siglo XIX que puso de manifiesto los quiebres de la argumentación pretendidamente universalista que mantenían las sufragistas blancas. Ella es quien introduce por primera vez la intersección entre "raza" y género. El simbolismo de esta mujer es importante porque critica esa reivindicada homogeneidad de las demandas de las mujeres. Se puede hacer una analogía en cuanto a lo que sucede con las mujeres con discapacidad. La lucha de las mujeres desde el siglo XIX, cuando las sufragistas exigían el derecho al voto, se fundamentaba en la denuncia de la domesticidad y el culto a la "verdadera feminidad", que evidentemente sólo afectaba a las mujeres blancas (y sin discapacidad). La crítica de Truth es relevante porque puede aplicarse también a las mujeres con discapacidades, aún cuando la intersección que trata de poner en evidencia sea otra, y haya transcurrido más de siglo y medio desde que estas palabras fueron pronunciadas: "ese hombre de allí dice que las mujeres necesitan ayuda al subirse a los carruajes, al cruzar las zanjas y que deben tener el mejor sitio en todas partes ipero a mí nadie me ayuda con los carruajes, ni a pasar sobre los charcos, ni me dejan un mejor sitio, ¿y acaso no soy yo una mujer?”3.

Este texto ha sido extraído del discurso Ain't I a woman? pronunciado por Truht en 1851 durante la Convención Nacional por los Derechos de Mujer celebrada en Akron, Ohio, EE.UU, publicado en Cózar Gutiérrez (2011: 28). 
Uno de los temas principales de los estudios de género a partir de los años 90 es precisamente el cuestionamiento del concepto homogéneo de "mujer", y las discusiones acerca de lo que engloba la identidad del sujeto femenino. En 1990 Judith Butler publica El género en disputa, que pone en cuestión algunos asuntos fundamentales de las teorías y de las prácticas feministas. La cuestión más importante que plantea Butler (2007), y que más repercusión ha tenido tanto en los debates teóricos como en la práctica política, es la pregunta acerca de cuál es el sujeto del feminismo. Del mismo modo, los estudios sobre la discapacidad van a cuestionarse la identidad del discapacitado y los términos que lo definen, así como la naturalización de la identidad del sujeto discapacitado. Además, la interseccionalidad que conforma la identidad subjetiva es un tema fundamental en la teoría sobre la discapacidad. Y por último, la corporalidad de los sujetos -tema prioritario en la crítica feminista-, es fundamental en los estudios sobre la discapacidad, dado que las marcas y las formas corporales son el primer signo de su discriminación, como ocurre con las mujeres. Por otra parte, frente a la naturalización de los diversos cuerpos -lo que provoca su discriminación y desvalorización-, el feminismo propugna la interpretación cultural y genealógica de los mismos, para así descubrir los mecanismos ideológicos que han permitido que tales figuras subjetivas hayan sido producidas.

El movimiento feminista y el de la discapacidad como corrientes críticas han conducido al desarrollo de importantes líneas de estudio. Una la constituye la investigación de las representaciones culturales de la discapacidad, donde podemos situar los estudios deconstructivistas históricos, el estudio de distintos pueblos y áreas geográficas, o el análisis de la imagen de la discapacidad a partir de la experiencia personal. Desde esta posición, se tienen en cuenta diversos factores diferenciales como edad, "raza", clase social, tipo de discapacidad, etc., que pueden tener implicaciones importantes en las identidades personales y ser significativos en las experiencias de personas con discapacidad (López González, 2007: 141). Cada vez más, los estudios de discapacidad incorporan esta orientación del estudio de la diversidad, tomando el enfoque metodológico y las herramientas de investigación feminista (Thomas, 1999). A pesar de algunos desencuentros, en la actualidad existen orientaciones confluentes por parte de las dos corrientes de análisis teórico y activismo político implicadas: feminismo y movimiento de la discapacidad. Precisamente ha sido el hecho de que existan personas que participan, como académicas y como activistas prácticas en ambas corrientes y movimientos, y que se vean implicadas personalmente en las cuestiones en debate (como mujeres y con discapacidades muchas de ellas), lo que está llevando a desarrollar nuevos enfoques de investigación y nuevas interpretaciones al abordar y reflejar la compleja y multifacética realidad que se trata: la que concierne a la vida de las mujeres con discapacidad (López González, 2007).

\section{A MODO DE CONCLUSIÓN}

La discapacidad es un tema que merece seguir teniendo una discusión formal y crítica a fin de rescatar la experiencia de vida y la cotidianidad de los sujetos catalogados como tales y considerados como "población vulnerable". Uno de los aspectos pertenecientes a dicha cotidianidad y experiencia de vida es el género, categoría que se construye histórica, sociocultural y políticamente, la cual influye de manera significativa en la subjetividad de los sujetos, háblese de mujeres y de hombres. 
Hablar de discapacidad implica, al igual que la categoría de género, aludir a una construcción social que ubica a unas personas en condiciones de superioridad sobre otras, por lo que se torna en relaciones de poder.

Las capacidades son facultades y aptitudes encarnadas en seres humanos. Por sí mismas constituyen la base de discursos a favor de la igualdad de oportunidades, la no discriminación, el reconocimiento de derechos para la inclusión o la plena participación social. Con los rasgos más visibles, la persona puede ser titular de derechos y deberes, o dejar de serlo, cuestión que se profundiza cuando se vive una situación de discapacidad física, sensorial, visual, auditiva, o mental, o cuando se experimentan situaciones de discapacidad múltiple (Munévar, 2003 Cit. en Ángela Díaz y Muñoz Borja, 2005: 163).

El proceso de construcción tiene implícita una dinámica social que históricamente ha incorporado unos imaginarios en el colectivo en torno a la discapacidad. Quien padece algún tipo de deficiencia estructural y/o funcional es considerada como una persona incompetente, inválida, descalificada, disminuida, inhabilitada, insuficiente, carente... todos éstos limitantes de la identidad personal, la interacción social y, por lo tanto, del desarrollo humano. Inevitablemente este sujeto sufrirá la discriminación y la exclusión de su contexto (Ángela Díaz y Muñoz Borja, 2005), y más si se trata de mujeres con discapacidades.

Hablar de mujeres con discapacidad significa aludir a un determinado "grupo". Puede significar ser "portadora" de una condición social de desventaja frente a "otro grupo". Y puede significar tener una representación social estigmatizada con efectos tangibles y materiales, y tener en sí misma un estigma, en el sentido que lo plantean autores como Erving Goffman (2001 [1963]). Este tipo de consideraciones conllevan consigo una determinada organización social en términos de diversos tipos de discriminación, mediante los cuales se reducen en la práctica las posibilidades de vida de las personas. Así, se construye una teoría del estigma, una ideología para explicar la inferioridad y dar cuenta del peligro que representan ese "tipo de personas" o colectivos. Entre otras consideraciones, hablar de mujeres con discapacidades hace referencia a la constitución de "identidades deterioradas" (Goffman, 2001 [1963]), o de condiciones de estigma donde se puede encontrar una marca, una seña que sirve para reconocer un defecto, una anomalía.

Entre la categoría género y la categoría discapacidad es posible identificar aspectos comunes como el "cuerpo marcado", las relaciones de poder y la sexualidad. Estas realidades conllevan consigo, como se viene argumentando a lo largo de este análisis, unas prácticas concretas de organización política y social con consecuencias inmediatas para la existencia de las personas. Es aquí donde cobra especial relevancia la relación entre identidad y ciudadanía. La discapacidad ubica en una situación de inferioridad, dependencia, vulnerabilidad y debilidad a quienes la viven, puesto que la sociedad considera que han perdido parte de su humanidad.

\section{REFERENCIAS BIBLIOGRÁFICAS}

Albrecht, G. L., Seelman, K. D. y Bury, M. (Eds.). (2001). Handbook of Disability Studies. Thousand Oaks, California: Sage.

Ángela Díaz, L. y Muñoz Borja, P. (2005). Implicaciones del género y la discapacidad en la 
construcción de identidad y la subjetividad. Revista Ciencias de la Salud, vol. 3, n. 2, 156-167.

Arnau Ripollés, S. (2004). Violencia de género contra la(s) mujer(es) con discapacidad(es).

Ponencia presentada en el Seminario: Violencia de género y discapacidades, Eraberri, Vitoria-Gasteiz.

Balza, I. (2011). Crítica feminista de la discapacidad: El monstruo como figura de la vulnerabilidad y exclusión. DILEMATA, vol. 3, n. 7, 57-76.

Barnes, C., Mercer, G. y Shakespeare, T. (1997). Exploring Disability. A Sociological Introduction. Cambridge: Polity Press.

Barton, L. (1998). Sociología y discapacidad: Algunos temas nuevos. En L. Barton (Comp.), Discapacidad y sociedad (pp. 19-33). Madrid: Morata.

Benedetti, M. (1974). Poemas de Otros. Buenos Aires: Editorial Alfa.

Bourdieu, P. (2007 [1998]). La dominación masculina. Barcelona: Anagrama. (1987). Cosas dichas. París: Les éditions de Minuit. (1991). El sentido práctico. Madrid: Taurus. (1992). Una invitación a la sociología reflexiva. Buenos Aires: Siglo XXI.

. y Wacquant, L. (1995). Respuestas por una antropología reflexiva. México D.F.: Grijalbo.

Bradley, E. (1995). The ICIDH: Format, Application in Different Settings and Distinction between Disability and Handicap. International Disability Studies, vol. 9, n. 3, 122-125.

Braidotti, R. (2005). Metamorfosis. Hacia una teoría materialista del devenir. Madrid: Akal.

Butler, J. (2007 [1990]). El género en disputa. El feminismo y la subversión de la identidad. Madrid: Piadós Studio.

Canguilhem, G. (2005 [1966]). Lo normal y lo patológico. Buenos Aires: Siglo XXI.

Corker, M. (1998). Deaf and Disabled or Deafness Disabled? Buckingham: Oxford University Press.

Cózar Gutiérrez, Mª Á. (Coord.). (2011). Autodiagnóstico de la situación de las mujeres con discapacidad en Andalucía. España: Dirección General de Personas con Discapacidad, Consejería para la Igualdad y Bienestar Social de la Junta de Andalucía.

Curiel, O. (2011). La descolonización vista desde el feminismo afro. En C. Villalba Augusto y N. Álvarez Lucena (Coords.), Cuerpos políticos y agencia. Reflexiones feministas sobre cuerpo, trabajo y colonialidad (pp. 197-212). Granada: Universidad de Granada.

Davis, L. J. (1997). The Disability Studies Reader. New York: Routledge.

Ferrante, C. y Ferreira, M. A. V. (2008). Cuerpo, discapacidad y trayectorias sociales: Dos estudios de caso comparados. Revista de Antropología Experimental, n. 8, 403-428.

Ferreira, M. A. V. (2010). De la minus-valía a la diversidad funcional: Un nuevo marco teóricometodológico. Política y Sociedad, vol. 47, n. 1, 45-65.

Foucault, M. (2009 [1979]). Vigilar y castigar. Nacimiento de la prisión. Madrid: Siglo XXI. (2009 [1978]). Historia de la sexualidad I. La voluntad del saber. Madrid: Siglo XXI.

Goffman, E. (2001 [1963]). Estigma. La identidad deteriorada. Buenos Aires: Amorrortu.

Hill Collins, P. (1998). Fighting Words: Black Women and the Search for Justice. Minneapolis: University of Minnesota Press.

Hooks, B. (2005). Racismo estético: Alisando nuestro pelo. La Gaceta de Cuba, n. 1, 70-73.

Huertas, R. (2011). En torno a la construcción social de la locura. Ian Hacking y la historia cultural de la psiquiatría. Revista de la Asociación Española de Neuropsiquiatría, vol. 31, n. 111, 437-456.

Ibáñez, J. (1992). Más allá de la sociología. El grupo de discusión: Técnica y crítica. Madrid: Siglo XXI.

Imrie, R. (1997). Rethinking the Relationships between Disability, Rehabilitation and Society. Disability and Rehabilitation, vol. 19, n. 7, 263-271.

Karsz, S. (2004). La exclusión, bordeando sus fronteras. Definiciones y matices. Barcelona: Gedisa.

López González, M. (2007). Discapacidad y género. Estudio etnográfico sobre mujeres 
discapacitadas. Educación y Diversidad. Revista Inter-universitaria de Investigación sobre Discapacidad e Interculturalidad, n. 1, 137-172.

Marks, D. (1999). Disability: Controversial Debates and Psychosocial Perspectives. London: Routledge.

(1997). Who Needs Models? Disability and Rehabilitation, vol. 19, n. 7, 492-495.

Martín Jorge, M. y Soriano Rovira, Ma. Á. (2008). Manual de sociología de la salud para el trabajo social. Granada: Elle Ediciones.

Moore, M., Beazley, S. y Maelzer, J. (1998). Researching Disability Issues. Buckingham: Open University Press.

Navarro, S. (2004). Redes sociales y construcción comunitaria. Creando (con)textos para una acción social ecológica. Madrid: Editorial CCS.

Oliver, M. (1998). ¿Una sociología de la discapacidad o una sociología discapacitada? En L. Barton (Comp.), Discapacidad y sociedad (pp. 34-58). Madrid. Morata. (1992). Changing the Social Relations of Research Production? Disability, Handicap and Society, vol. 7, n. 2, 101-114. . (1990). The Politics of Disablement. Basingstoke: McMillan.

Parsons, T. (1982 [1951]). El sistema social. Madrid: Alianza.

Rodríguez, J. A. (1987). Salud y sociedad. Análisis sociológico de la estructura y la dinámica del sector sanitario español. Madrid: Tecnos.

Rodríguez Díaz, S. y Ferreira, M. A.V. (2010). Desde la Dis-Capacidad hacia la diversidad funcional. Un ejercicio de Dis-Normalización. Revista Internacional de Sociología (RIS), vol. 68, n. 2, 289-309.

Rosato, A., Angelino, A., Almeida, M. E., Angelino, C., Kippen, E., Sánchez, C., Spadillero, A., Vallejos, I., Zuttión, B. y Priolo, M. (2009). El papel de la ideología de la normalidad en la producción de discapacidad. Ciencia, Docencia y Tecnología, n. 39, 87-105.

Rosenberg, E. (1989). Disease in History: Frames and Framers. Milibank Quarterly, vol. 67, sup. 1, 1-15.

Shakespeare, T. (1998). Poder y prejuicio: Los temas de género, sexualidad y discapacidad. En L. Barton (Comp.), Discapacidad y sociedad (pp. 205-229). Madrid: Morata.

Shum, G. y Conde, Á. (2009). Género y discapacidad como moduladores de la identidad. Feminismo/s: Revista del Centro de Estudios sobre la Mujer, n. 13, 119-132.

Thomas, C. (1999). Female Forms: Experiencing and Understanding Disability. Buckingham: Open University Press.

Thomson, R. G. (2005). Feminist Disability Studies. Signs: Journal of Women in Culture and Society, vol. 30, n. 2, 1557-1587.

Torres Dávila, Ma . S. (2004). Género y discapacidad: Más allá del sentido de la maternidad diferente. Maestría en Estudios de Género. FLACSO: Sede Ecuador.

Wright, S. (1998). La politización de la cultura. Anthropology Today, vol. 14, n. 1, 1-19. 
